\title{
A REVIEW ON “DRY SYRUPS FOR PAEDIATRICS”
}

\author{
P. S. BHANDARE 1 , A. V YADAV 2
}

${ }^{*}$ Department of Pharmacology, GIPER, Limb, Satara: 415015, India, ${ }^{2}$ Principal, GIPER, Limb, Satara: 415015, India Email: pooja14511@gmail.com

Received: 10 Oct 2016, Revised and Accepted: 20 Dec 2016

\begin{abstract}
Suspensions may be defined as preparations containing finely divided drug particles (the suspensoid) distributed somewhat uniformly throughout a vehicle with or without stabilizers and other additives in which drug exhibits a minimum degree of solubility hence conventional oral suspension can be administered immediately (ready to use form) and not requiring reconstitution at the time of dispensing are simply designated as "Oral Suspension".

There is an important category of suspension that are available as dry powders intended for suspension in liquid vehicles. These are dry mixtures containing the drug and suitable suspending and dispersing agents to be diluted and agitated with a specific quantity of vehicle, most often purified water. Drugs that are instable if maintained for extended periods in the presence of aqueous vehicle (eg., many antibiotic drugs) are frequently supplied as dry powder mixtures for reconstitution at the time of dispensing. This type of preparation is designated in the USP by a title "for Oral Suspension". The reconstituted system is the formulation of choice when the drug stability is a major concern. After reconstitution, these systems have a short but acceptable life if stored at refrigerator temperatures. Reconstitutable oral systems show the adequate chemical stability of the drug during shelf life, avoids the physical stability problems related to solubility, $\mathrm{pH}$ and incompatibilities with other ingredients and also reduce the weight of the final product because the aqueous vehicle is absent and consequently the transportation expenses may be reduced.
\end{abstract}

Dry syrup form of the drug is also useful in case of bioavailability as it has high bioavailability rather than tablets and capsules as it disintegrates in water outside of the oral cavity and directly the suspension is gone through the gastrointestinal tract. So the suspension easily absorbs in the GIT.

A number of commercial and official preparations are available as dry powder mixtures. The present review gives an account of the excipients used, methods of preparation of dry syrups along with their evaluations, their packaging, examples of research articles, few marketed preparations.

Keywords: Dry suspension, Dry syrup, Reconstitutable oral suspension. Patient compliance and stability of formulation

(C) 2016 The Authors. Published by Innovare Academic Sciences Pvt Ltd. This is an open access article under the CC BY license (http://creativecommons.org/licenses/by/4.0/] DOI: http://dx.doi.org/10.22159/ijcpr.2017v9i1.16789

\section{INTRODUCTION}

Many antibiotic materials are unstable when maintained in solution for an appreciable length of time, and therefore, from a stability standpoint, insoluble forms of the drug substances in aqueous suspensions or as dry powder for reconstitution are attractive to manufacturers [1]. Since decades among all the pharmaceutical products available, oral drug delivery has gained a higher scope and popularity and has been widely employed for the systemic delivery of drugs. The positive aspect regarding the oral dosage form which created its high level of acceptance was its ease of administration, patient compliance and stability of formulation [2]. The antibacterial oral suspensions include preparations of antibiotics substances (eg., erythromycin derivatives, and tetracyclines and its derivatives), sulfonamides (eg., sulfamethoxazole and sulfisoxazole acetyl), other anti-infective agents (eg., methenamine mandelate and nitrofurantoin), or combinations of these (eg., sulfamethoxazole-trimethoprim). The antibiotic oral suspension, including those prepared by reconstitution, provide a convenient way to administer dosages to infants and children and to adult patients who prefer liquid preparations to solid ones. Although studies have demonstrated that the dry oral suspension after constitution in a liquid is stable for $24 \mathrm{~h}$ after preparation, reconstituted solution remains stable when stored in the refrigerator for the labelled period, usually 7 to $14 \mathrm{~d}$, depending on the preparation. This is a sufficient period for the patient to complete the regimen usually prescribed. However, in case the medication remains after the patient completes the course of therapy, the patient should be instructed to discard the remaining portion, which would be unfit for use at the later time [1].

Examples of dry powders mixtures intended for reconstitution to oral solutions are as follows:

i) Cloxacillin Sodium for Oral Solution, USP (Teva), an anti-infective antibiotic. ii) Penicillin V Potassium for Oral Solution, USP (Veetids, Geneva), an anti-infective antibiotic.

iii) Potassium Chloride for Oral Solution, USP (K-LOR, Abbott), a potassium supplement.

\section{Disadvantages of liquid oral suspensions $[1,4,5]$}

- It is a bulk formulation, so there are chances of inaccuracy in single dosing.

- Drug dose depends on various physical factors of the dosage form such as the temperature of storage, sedimentation rate of the formulation, liquid flow properties like viscosity, pourability, redispersion, flocculation and content uniformity.

- Stability of the liquid suspension largely depends on the temperature of storage.

- Caking occurs upon storage.

Advantages of dry granules for oral suspension $[1,4,5]$

- There is accurate single dosing as the dose is packed in single dose sachets.

- Drug dose is comparatively independent of any physical factors like temperature, sedimentation rate and liquid flow properties.

- The packaging of the powder mixture is done in sachets making the formulation easy to carry.

- The enhanced convenience of the single dosage regimen.

- Colored, flavored, sweetened formulation is advantageous for administration to the paediatric population. 
- Stable on storage and when reconstituted with an ingestible liquid for administration, the corresponding liquid suspension is stable for the duration for which the therapy is required.

\section{Reasons for formulation of such suspensions $[1,4,5]$}

The most common reason for the formulation of suspensions for reconstitution is the inadequate chemical stability of the drug in an aqueous vehicle. In such cases, dissolution or even suspension of the drug results in a very short shelf life. For example, reconstituted suspensions of penicillin have a maximum shelf life of $14 \mathrm{~d}$. The manufactured dry mixture, however, has a shelf life of at least $2 \mathrm{y}$.

Another reason for the formulating suspensions for reconstitution is to avoid the physical stability problems often encountered in conventional suspensions. These problems include possible increased drug solubility due to $\mathrm{pH}$ changes from chemical degradation, incompatibility of ingredients, viscosity changes, conversion of polymorphic form and crystal growth and caking.

Formulation for reconstitution reduces the weight of the final product because the aqueous vehicle is absent and consequently, transportation expenses may be reduced. The dry mixture may be shipped without regard to seasonal temperatures because its physical stability is less susceptible to temperature extremes as compared with conventional suspensions.

\section{Desired attributes}

During manufacture, the dry blend, or mixture, must be a uniform mixture of the appropriate concentration of each ingredient. It must not segregate into a non-uniform mix because errors in dosage may result. During reconstitution, the powder blend must disperse quickly and completely in the aqueous vehicle. The reconstituted suspension must be easily redispersed and poured by the patient to provide an accurate and uniform dose. After reconstitution, the high viscosity caused by the refrigerated storage temperatures should not obstruct dose administration by the patient. The final product must have an acceptable appearance, taste, odor.

\section{Excipients used $[4,5]$}

The criteria for selecting excipients are based both on suitability for reconstitution and on the physical type of powder mixture desired.

- A number of excipients should be kept to a minimum as more the number of excipients in the formulation, the greater is the possibility of problems, for example, the chances of compatibility problems are increased as more excipients are used. Greater processing is required to incorporate more excipients. More excipients will require sampling and testing for quality control. A common method of reducing the number of excipients is to use an excipient that performs more than one function. Eg. Sucrose can be used as diluents, sweetener and suspending agent.

- All excipients should disperse rapidly on reconstitution. This criterion eliminates several suspending agents.

- Many preservatives are also not suitable.

\begin{tabular}{ll}
\hline Frequent & Infrequent \\
\hline Suspending agent & Anticaking agent \\
Wetting agent & Flocculating agent \\
Sweetener & Granule disintegrant \\
Preservative & Granule binder \\
Color & Lubricant \\
Flavor & Solid diluents \\
Buffer & Antioxidant \\
\hline
\end{tabular}

\section{Granule disintegrant}

It results in prevention of the particle aggregation. The swelling of disintegrant grains in water plays an important part in the deaggregation of drug particles.

\section{Granule binder}

It helps to reduce the settling of particles in suspensions. It is also used as a stabiliser for suspensions. eg. High molecular weight povidone.

\section{Suspending agents}

Suspending agents should be easily dispersed by vigorous hand shaking during reconstitution. This rules out several common suspending agents because many require hydration, elevated temperatures or high shear mixing for adequate dispersion. Some of the suspending agents that are recommended for use are Acacia, Carboxymethylcellulose sodium, Iota carrageenan, microcrystalline cellulose with carboxymethylcellulose sodium, Povidone, Propylene glycol alginate, Silicon dioxide, Sodium starch glycolate, Tragacanth, Xanthan gum.

The combination of microcrystalline cellulose and sodium carboxymethylcellulose is a common suspending agent. Total concentrations of the combination greater than $1 \%$ in the reconstituted product can result in thyrotrophic gels. This agent and sodium carboxymethylcellulose alone are anionic; they are incompatible with many cationic excipients.

The natural gums are usually anionic and include exudates of tree and extract from seaweed. Acacia and tragacanth have been used as suspending agents for many years. The carrageenan and alginate suspending agents are seaweed extracts. The alginates produce highly viscous solutions, and the iota-carrageenans produce thixotropic dispersions. A disadvantage of these natural products is batch variation in colour, viscosity, gel strength and hydration rate.

Xanthan gum is a common suspending agent in suspensions for reconstitution. Because it is produced by microbial fermentation, there is good batch-to-batch uniformity and few microbial problems. Its solution viscosity is practically independent of $\mathrm{pH}$ and temperature $[1,4,5]$.

\section{Sweeteners [1, 4-5]}

The sweetener is a significant component of suspensions for reconstitution. Drugs frequently have a bitter taste and suspending agents; particularly clays may have a bland taste. Sweeteners can mask the unfavourable taste and enhance patient acceptance in the paediatric population that uses this produce. An increased viscosity as a result of the sweetener aids suspension of the drug particles. The sweeteners used are Sucrose, Mannitol, Aspartame, Sodium saccharin, Dextrose.

Sucrose can perform both functions of sweetener and suspending agent and can serve as diluents in the dry mixture. Aspartame has fair acid stability but poor heat stability. Saccharin may become restricted by the Food and Drug Administration because of its carcinogenic potential $[4,5]$ Addition of saccharin or its salts is not permitted in the preparations meant for paediatric use [8].

\section{Wetting agents $[4,5]$}

Many drugs in suspension are hydrophobic, they repel water and are not easily wetted. Surfactants are commonly used to aid in the dispersion of hydrophobic drugs. The formulator must select the appropriate wetting agent for optimum dispersion of the drug at the lowest effective concentrations. The excess wetting agent can produce foaming and impart an unpleasant taste.

Polysorbate 80 is a common wetting agent. It is nonionic and is chemically compatible with both cationic and anionic excipients and drugs. It is used in concentrations lesser than or equal to $0.1 \%$. Another common wetting agent is Sodium lauryl sulfate. This agent is anionic and may be incompatible with cationic drugs.

\section{Other excipients [1, 4-5]}

The other excipients include buffers, preservatives, flavours and colours. Flocculating agents are not commonly used in suspensions for reconstitution because these products are usually redispersed frequently enough to prevent caking.

Buffers are used to maintain the optimum $\mathrm{pH}$ for all excipients. Suspension $\mathrm{pH}$ is often adjusted to ensure that the drug remains insoluble. The polymeric suspending agents, however, have the greatest viscosity at the $\mathrm{pH}$ of their maximum solubility. Sodium citrate is a common buffer used in suspensions for reconstitution.

Preservatives are required in most suspensions because the suspending agents and sweetener are often good growth media for 
microorganisms. The choice of preservatives is limited because most of these ingredients require extended time periods for dissolution at room temperatures. eg., sorbic acid. Sucrose in sufficient concentrations (ca.60\%w/w) can aid in the prevention of microbial growth. Other common preservatives used are Sodium benzoate and Sodium propionate.

Flavours enhance patient acceptability of the product. Both natural and artificial flavours are used. Additional flavours used include raspberry, pineapple, etc. In some cases, refrigeration after reconstitution is required for the stability of the flavouring agent rather than for the stability of the drug.

Colourants are intended to provide a more aesthetic appearance to the final suspension. As relatively large cations or anions, these agents may be chemically incompatible with other ingredients. For example FD and C Red No.3 is a disodium salt, is anionic and would be incompatible with a cationic wetting agent.

Anticaking agents such as amorphous silica gel have several functions in suspensions for reconstitution. A common problem in dry mixtures is poor powder flow and caking. This is often caused by powder agglomeration due to moisture uptake. As a desiccant, these agents remove moisture from the dry mixture to facilitate good powder flow and prevent caking. In addition, anticaking agents separate the dry particles to inhibit fusion

\section{Preparation of dry mixture $[1,4-5]$}

- Powder blends

- Granulated products

- Combination products

\section{Powder blends}

Powder blends, sometimes called powder mixtures are prepared by mixing the excipients of the dry mixture in powder form. Excipients present in small quantities may require a two stage mixing operation. Such excipients can be mixed with a portion of a major excipient to aid in their dispersion. For example, milled sucrose provides a large surface area for the adsorption of the small quantities of flavor oils. The second stage comprises the mixing of the remaining excipients. The selection of the appropriate mixer involves several considerations, the most significant of which is that the mixer should rapidly and reliably produce a homogenous mixture.

\section{Advantages}

- Least capital equipment and energy.

- Least likely to have chemical and physical stability problems because no heat or solvents are used.

- Low moisture content can be achieved in dry mixture.

\section{Disadvantages $[4,5]$}

- Prone to homogeneity problems. Two most important properties for the mixing of these powders are Particle size and Powder flow.

- Loss of the active ingredient during mixing.

- The loss during mixing is significant if potent drug which is used in very low concentrations is lost.

\section{Combination product}

Powdered and granulated excipients can be combined to overcome some disadvantages of granulated products. Less energy and equipment for granulation may be required if the majority of the diluents can be added after granulation. Also, heat sensitive excipients such as flavours can be added after drying of the granulation to avoid exposure to elevated temperatures.

The general method is first to granulate some of the excipients, then blend the remaining excipients with the dried granules before filling the container. The presence of the diluents helps to improve flow and reduces both segregation and dust formation.

\section{Disadvantages $[4,5]$}

- Risk of non-uniformity

- Particle sizes of various fractions should be carefully controlled.

Processing the dry mixture $[4,5]$

- Use efficient mixing

- Determine an adequate duration of mixing time.

- Avoid accumulation of heat and moisture during mixing.

- Limit temperature/humidity variations. A general rule is $700 \mathrm{C}$ at $<40 \%$ relative humidity.

- The finished batch should be protected from moisture. Store in lined containers with silica desiccant bags.

- The sample for batch uniformity. Test at the top, middle and bottom levels of the dry mixture.

\section{Evaluation of oral reconstitute system [7]}

Eg: Cephalexin monohydrate with piperine re-constitutional oral suspension

\section{Drug content}

One dose ( $3.4 \mathrm{~g}$ of the formulation to $5 \mathrm{ml}$ ) is equivalent to $0.125 \mathrm{~g}$ of Cephalexin. The drug was extracted with $100 \mathrm{ml}$ of distilled water and the solution was filtered through nylon filter membrane $(0.22$ $\mu \mathrm{m}) .0 .1 \mathrm{ml}$ of the solution was further diluted to $10 \mathrm{ml}$ with distilled water and the absorbance of the solution was read at $\lambda \max 260 \mathrm{~nm}$ on Hitachi U-2800 UV spectrophotometer. The drug concentration was extrapolated from the calibration curve in distilled water.

\section{pH}

The $\mathrm{pH}$ of the reconstituted suspension was determined using a $\mathrm{pH}$ meter-Systronic $\mu \mathrm{pH}$ system 361. A glass rod was dipped into a suspension containing $100 \mathrm{mg}$ of drug filled in a $50 \mathrm{ml}$ of the beaker.

\section{Viscosity}

The rheologic parameters of the prepared suspensions, in terms of viscosity, were determined by use of the steady shear method, measuring the "non-Newtonian viscosity". Rheology of all suspensions was performed with a RVT Brookfield viscometer from Choksi Lab. (Indore, M. P.) All measurements were performed after eliminating all thixotropy from the suspension.

\section{Sedimentation volume ratio}

To study sedimentation of our suspensions, the sedimentation volume was determined as a function of time. The sedimentation volume $\mathrm{F}$ is defined as the ratio of the final, equilibrium volume of the sediment, $\mathrm{Vu}$ to the total volume $\mathrm{V} 0$ before settling, as expressed in the following equation:

\section{$\mathbf{F}=[\mathrm{Vu} / \mathrm{V0}]$}

In this study, the sedimentation volume was determined as a function of time. The suspension (height $=9 \mathrm{~cm}$ ) was decanted in a cylinder of $100 \mathrm{ml}$ with a diameter of $2.5 \mathrm{~cm}$. After $1 \mathrm{~h}, 24 \mathrm{~h}$ and $1 \mathrm{w}$, the sedimentation volume $\mathrm{F}$ was determined.

\section{In vitro drug release}

The in vitro dissolution studies were carried out using USP apparatus Type II at $100 \mathrm{rpm}$. The dissolution medium consisted of $900 \mathrm{ml}$ distilled water maintained at $370 \mathrm{C} \pm 0.50 \mathrm{C}$ the drug release at different time intervals was measured for two hours using Hitachi U2800 UV spectrophotometer.

ICH guidelines (q6a) for re-considerable oral suspensions $[4,6]$

\section{Oral liquids}

One or more of the following specific tests will normally be applicable to oral liquids and to powders intended for reconstitution as oral liquids. 


\section{a) Uniformity of dosage units}

This term includes both the mass of the dosage form and the content of the active substance in the dosage form; a pharmacopoeial procedure should be used. In general, the specification should include one or the other but not both. When weight variation is applied for new drug products exceeding the threshold value to allow testing uniformity by weight variation, applicants should verify during drug development that the homogeneity of the product is adequate. If appropriate, tests may be performed in-process; however, the acceptance criteria should be included in the specification. This concept may be applied to both single dose and multiple dose packages. The dosage unit is considered to be the typical dose taken by the patient. If the actual unit dose, as taken by the patient, is controlled, it may either be measured directly or calculated, based on the total measured weight or volume of drug divided by the total number of doses expected. If dispensing equipment (such as medicine droppers or dropper tips for bottles) is an integral part of the packaging, this equipment should be used to measure the dose. Otherwise, a standard volume measure should be used. The dispensing equipment to be used is normally determined during development.

For powders for reconstitution, uniformity of mass testing is generally considered acceptable.

\section{b) $\mathrm{pH}$}

Acceptance criteria for $\mathrm{pH}$ should be provided where applicable and the proposed range justified.

\section{c) Microbial limits}

Microbial limit testing is seen as an attribute of Good Manufacturing Practice, as well as of quality assurance. In general, it is advisable to test the drug product unless its components are tested before manufacture and the manufacturing process is known, through validation studies, not to carry a significant risk of microbial contamination or proliferation. It should be noted that, whereas this Guideline does not directly address excipients, the principles discussed here may be applicable to excipients as well as to new drug products. Skip testing may be an appropriate approach in both cases where permissible.

With acceptable scientific justification, it may be possible to propose no microbial limit testing for powders intended for reconstitution as oral liquids. Acceptance criteria should be set for the total count of aerobic microorganisms, total count of yeasts and molds, and the absence of specific objectionable bacteria (e. g., Staphylococcusaureus, Escherichia coli, Salmonella, Pseudomonas aeruginosa). These should be determined by suitable procedures, using pharmacopoeial procedures, and at a sampling frequency or time point in manufacture which is justified by data and experience.

\section{d) Antimicrobial preservative content}

For oral liquids needing an antimicrobial preservative, acceptance criteria for preservative content should be established. Acceptance criteria for preservative content should be based upon the levels of antimicrobial preservative necessary to maintain microbiological quality of the product at all stages throughout its proposed usage and shelf-life. The lowest specified concentration of antimicrobial preservative should be demonstrated to be effective in controlling microorganisms by using a pharmacopoeial antimicrobial preservative effectiveness test. Testing for antimicrobial preservative content should normally be performed at release, under certain circumstances, in-process testing may suffice in lieu of release testing. When antimicrobial preservative content testing is performed as an inprocess test; the acceptance criteria should remain part of the specification. Antimicrobial preservative effectiveness should be demonstrated during development, during scaleup, and throughout the shelf-life (e. g., instability testing).

\section{e) Antioxidant preservative content}

Release testing for antioxidant content should normally be performed. Under certain circumstances, where justified by developmental and stability data, shelf-life testing may be unnecessary, and in-process testing may suffice in lieu of release testing where permitted. When antioxidant content testing is performed as an in-process test, the acceptance criteria should remain part of the specification. If only release testing is performed, this decision should be reinvestigated whenever either the manufacturing procedure or the container/closure system changes.

\section{f) Extractables}

Generally, where development and stability data show evidence that extractable from the container/closure systems are consistently below levels that are demonstrated to be acceptable and safe, elimination of this test can normally be accepted.

This should be reinvestigated if the container/closure system or formulation changes.

Where data demonstrate the need, tests and acceptance criteria for extractable from the container/closure system components (e. g., rubber stopper, cap liner, plastic bottle, etc.) are considered appropriate for oral solutions packaged in non-glass systems, or in glass containers with non-glass closures. The container/closure components should be listed, and data collected for these components as early in the development process as possible.

\section{g) Alcohol content}

Where it is declared quantitatively on the label in accordance with pertinent regulations, the alcohol content should be specified. It may be assayed or calculated.

\section{h) Dissolution}

In addition to the attributes recommended immediately above, it may be appropriate (e. g., insoluble drug substance) to include dissolution testing and acceptance criteria for oral suspensions and dry powder products for resuspension. Dissolution testing should be performed at release. This test may be performed as an in-process test when justified by product development data. The testing apparatus, media, and conditions should be pharmacopoeial, if possible, or otherwise justified. Dissolution procedures using either pharmacopoeial or non-pharmacopoeial apparatus or conditions should be validated.

Single-point measurements are normally considered suitable for immediate-release dosage forms. Multiple-point sampling, at appropriate intervals, should be performed for modified release dosage forms. Acceptance criteria should be set based on the observed range of variation and should take into account the dissolution profiles of the batches that showed acceptable performance in vivo. Developmental data should be considered when determining the need for either a dissolution procedure or a particle size distribution procedure.

\section{i) Particle size distribution}

Quantitative acceptance criteria and a procedure for determination of particle size distribution may be appropriate for oral suspensions.

Developmental data should be considered when determining the need for either a dissolution procedure or a particle size distribution procedure for these formulations. Particle size distribution testing should be performed at release. It may be performed as an inprocess test when justified by product development data. If these products have been demonstrated during development to have consistently rapid drug release characteristics, exclusion of a particle size distribution test from the specification may be proposed. Particle size distribution testing may also be proposed in place of dissolution testing; justification should be provided. The acceptance criteria should include acceptable particle size distribution in terms of the percent of total particles in given size ranges. The mean, upper, and/or lower particle size limits should be well defined.

Acceptance criteria should be set based on the observed range of variation and should take into account the dissolution profiles of the batches that showed acceptable performance in vivo, as well as the intended use of the product. The potential for particle growth should 
be investigated during product development; the acceptance criteria should take the results of these studies into account.

\section{j) Redispersibility}

For oral suspensions which settle on storage (produce sediment), acceptance criteria for re-dispersibility may be appropriate. Shaking may be an appropriate procedure. The procedure (mechanical or manual) should be indicated. The time required to achieve resuspension by the indicated procedure should be clearly defined. Data generated during product development may be sufficient to justify skip lot testing or elimination of this attribute from the specification may be proposed.

\section{k) Rheological properties}

For relatively viscous solutions or suspensions, it may be appropriate to include rheological properties (viscosity/specific gravity) in the specification.

The test and acceptance criteria should be stated. Data generated during product development may be sufficient to justify skip lot testing or elimination of this attribute from the specification may be proposed.

\section{1) Reconstitution time}

Acceptance criteria for reconstitution time should be provided for dry powder products which require reconstitution. The choice of diluent should be justified. Data generated during product development may be sufficient to justify skip lot testing or elimination of this attribute from the specification may be proposed.

\section{m) Water content}

For oral products requiring reconstitution, a test and acceptance criterion for water content should be proposed when appropriate. Loss on drying is generally considered sufficient if the effect of absorbed moisture vs. water of hydration has been adequately characterised during the development of the product. In certain cases, a more specific procedure (e. g., Karl Fischer titration) may be preferable

\section{Packaging and storage $[1,3,8]$}

1. The dry powders for reconstitution should be packaged insufficiently wide mouth container having adequate air space above the liquid to facilitate the flow of contents.

2. The dry powders should be stored in a tight container protected from moisture, freezing, excessive heat and light.

3. The label should contain the direction stating: "Shake before Use" to ensure uniform distribution of solid particles and thereby to obtain uniform and proper dosage.

4. The dry powders should be stored at room temperature.

5. After reconstitution, the suspension should be stored in the refrigerator (freezing should be avoided to prevent aggregation)
6. For single dosage packing, sachets are used made up of 4 layers of aluminium foil.

7. With multiple dose powders, it may be necessary to provide a measuring device capable of delivering the quantity prescribed.

8. They are administered either in volumes of $5 \mathrm{ml}$. Each dose of multiple doses orally administered by means of a suitable measuring device which is usually provided in the container.

\section{Labelling [1, 8]}

- That the contents are meant for preparation of an oral liquid.

- The directions for preparing the oral liquid including nature and quantity of liquid to be used.

- The conditions under which the reconstituted solution should be stored.

- The period during which the constituted oral liquid may be expected to remain satisfactory for use when prepared and stored in accordance with manufacturer's recommendations.

- The strength in terms of active ingredients in a a suitable dose volume of reconstituted preparation.

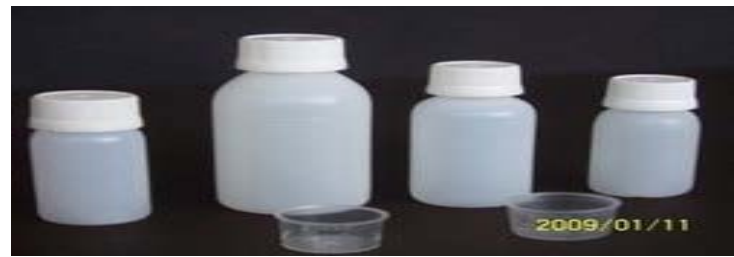

Directions for reconstitution $[1,8]$

i) When called on to reconstitute and dispense one of these products, the pharmacist loosens the powder at the bottom of the container by lightly tapping it against a hard surface and then add the label designated amount of purified water, usually in portions, and shake until all the dry powder has been suspended.

ii) It is important to add precisely the prescribed amount of purified water to the dry mixture if the proper drug concentration per dosage unit is to be achieved.

iii) Also, the use of purified water rather than tap water is needed to avoid the possibility of adding impurities that could adversely affect the stability of the resulting preparation.

iv) Generally, manufacturers provide the dry powder or granule mixture in slightly oversized containers to permit adequate shakinf of the contents after the entire amount of purified water has been added.

v) The pharmacist should not "eyeball" the amount of water to be added or fill up the bottle with purified water.

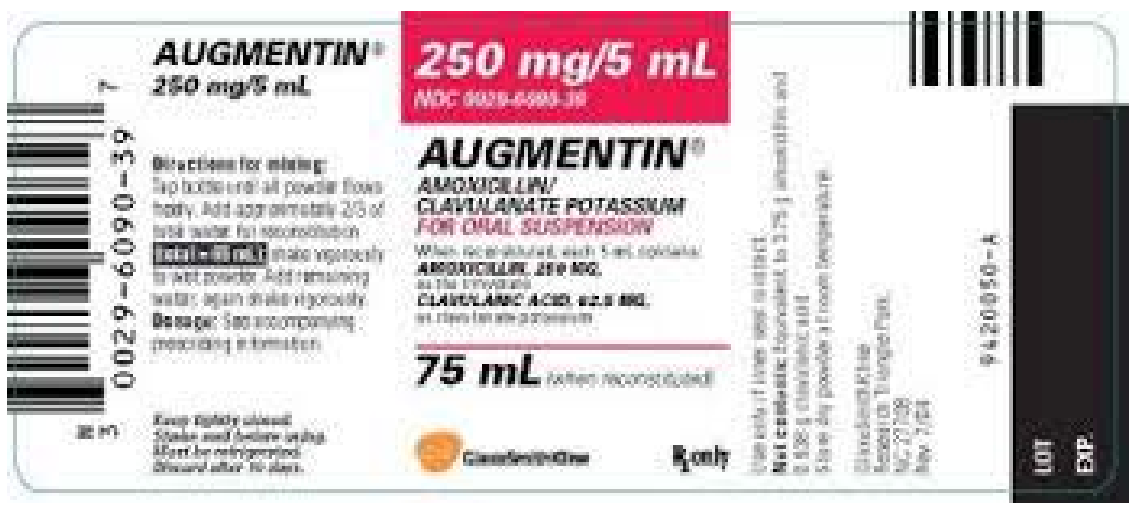

Fig. 1: Augmentin for oral suspension label 
Table 1: List of marketed preparations

\begin{tabular}{|c|c|c|}
\hline S. No. & Name & Drugs \\
\hline 1. & Augmentin DS & Clavulanic acid, amoxicillin \\
\hline 2. & Clavum DS & \\
\hline 3. & Droclav DS & \\
\hline 4. & Estamox CV dry syrup & \\
\hline 5. & Moximax CV dry syrup & \\
\hline 6. & Abi-CV & \\
\hline 7. & Moxyrad-CL dry syrup & \\
\hline 8. & Advance dry syrup & \\
\hline 9. & Volmox-CV dry syrup & \\
\hline 10. & MOCA dry syrup & \\
\hline 1. & Phexin DS & Cephalexin \\
\hline 2. & Enceff dry syrup' & \\
\hline 1. & Nupod DS & Cefpodoxime \\
\hline 2. & Podoxrad dry syrup & \\
\hline 3. & Estidox dry syrup & \\
\hline 4. & Atmocef dry syrup & \\
\hline \multirow[t]{4}{*}{5.} & Spinxo dry syrup & \\
\hline & Kefloxin DS & Cefadroxil \\
\hline & Flemoxin DS & Amoxicillin \\
\hline & Betaclox 125 dry syrup & Dicloxacillin \\
\hline 1. & Cefixrad DS & Cefixime \\
\hline 2. & Linicef dry syrup & \\
\hline 3. & Lofixim dry syrup & \\
\hline 4. & Sectocef $100 \mathrm{DS}$ & \\
\hline \multirow[t]{6}{*}{5.} & Taxim- 0 dry syrup & \\
\hline & Necfix-O dry syrup & Cefixime and Ofloxacin \\
\hline & Nuzylac forte dry syrup & Lactobacillus and enzyme fortified with B-complex and simethicone \\
\hline & Satrogyl-0 dry syrup & Satrranidazole \\
\hline & & Ofloxacin \\
\hline & Dryvit LB dry syrup & Vitamin B complex with probiotic dry syrup \\
\hline 1. & Znsol-P dry syrup & Zinc Gluconate with prebiotic-probiotic oral suspension. \\
\hline \multirow[t]{3}{*}{2.} & Gutcon dry syrup & \\
\hline & Azimax 200 dry syrup & Azithromycin \\
\hline & Flucamed powder for oral suspension & Fluconazole \\
\hline
\end{tabular}

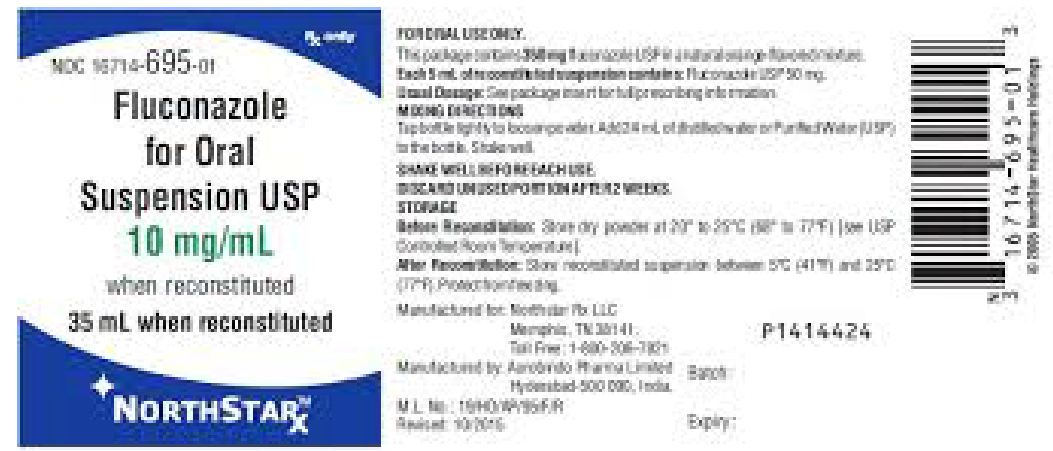

Fig. 2: Fluconazole for oral suspension label

\section{Review article $[9,10]$}

- Development of re-constitutable suspensions containing diclofenac sodium-loaded microspheres for pediatric delivery [9]

Effective clinical utilisation of non-steroidal anti-inflammatory drugs, such as diclofenac sodium (DS) is significantly limited by their ulcerogenic potential and poor bioavailability after oral administration. The objective of this work was to develop reconstitutable paediatric suspensions of DS-loaded microspheres prepared with an acrylic polymer (Eudragit RS) for improved paediatric delivery of DS. The results of preliminary characterisation studies of suspensions show that a liquid pharmaceutical preparation for oral administration capable of providing a sustained release of DS was successfully obtained.
- A new reconstitutable oral paediatric hydrocortisone solution containing hydroxypropyl- $\beta$-cyclodextrin $[4,10]$

Hydrocortisone (HC) despite a low aqueous solubility and a very poor palatability is frequently used unlicensed in paediatric practice. Hence a reconstitutable taste masked hydrocortisone solution with the potential to be easily produced extemporaneously was developed. Excipients for the reconstitutable dry powder mix were selected based on their aqueous solubility, compatibility, safety profile in children and stability at the optimum $\mathrm{pH}$ for HC. Formulations were visually inspected, and $\mathrm{pH}$ was checked. The microbiological assessment showed that the selected preservative combination was efficient and the presence of preservative ensured the recommended acceptance criteria for microbiological quality after reconstitution with repetitive sampling. The successfully developed $\mathrm{ng} / \mathrm{ml}$ reconstituted oral 
palatable paediatric $\mathrm{HC}$ solution was stable for $1 \mathrm{mo}$ after reconstitution and has the potential to facilitate dosing, acceptability, availability and affordability.

\section{CONCLUSION}

The reconstituted oral suspension shows high levels of acceptance in case of ease of administration, patient compliance and physical stability I reduced compared with that of conventional suspensions. It also reduces the weight of the final product because the aqueous vehicle is absent and consequently, transportation expenses may be reduced. The dry mixture may be shipped without regard to seasonal temperatures. Therefore this is an ideal formulation for paediatrics for the administration of mainly the antibiotic drugs if constituted as per the directions mentioned.

\section{ACKNOWLEDGEMENT}

The authors would like to express gratitude to Librarian and the supporting staff for providing necessary book, internet facilities to carry out this research work.

\section{CONFLICT OF INTERESTS}

Declared none

\section{REFERENCES}

1. Howard CA. Introduction of pharmaceutical dosage forms; Lea and Febiger, Philadelphia; 1981. p. 139-66.

2. Ofner CM, Schnaare RL, Schwartz JB. Reconstitutable oral suspension". Lieberman HA, Rieger MM, Banker GS. Pharmaceutical dosage forms and disperse system; Vol. 2. $2^{\text {nd }}$ Edition. (New York: Marcel dekkar Inc) NJ; 1984. p. 243-59.
3. Lieberman HA, Rieger MM, Banker GS. Pharmaceutical dosage forms and disperse system. Vol. 2. $2^{\text {nd }}$ Edition. (New York: Marcel dekkar Inc.); 1994. p. 149-77.

4. Casilda Bardeskar, Racheal Geeverghese. Reconstituitable oral suspensions (Dry Syrups): an overview. World J Pharm Res 2015;4:462-84

5. Parmar Pratik, MR Patel, KR Patel, NM Patel. "A review on taste masking pediatric dry syrup". Int J Univers Pharm Bio Sci 2013;2:2319-8141.

6. European Medicines Agency ICH Topic Q 6 A Specifications: test procedures and acceptance criteria for new drug substances and new drug products: Chemical Substances; 2000.

7. Vijay Kumar Singh, Vikash Kumar Mishra, Jayant Kumar Maurya. Formulation and evaluation of cephalexin monohydrate re-constitutional oral suspension with piperine and their antibacterial activity. World J Pharm Res 2014; 3:821-31.

8. Indian pharmacopoeia. Vol. II; 2014. p. 952-1057.

9. Umut Can Oz, Burcu Devrim, Asuman Bozkır, Kandemir Canefe. Development of reconstitutable suspensions containing diclofenac sodium-loaded microspheres for pediatric delivery. J Microencapsulation 2015;32:317-28.

10. Mine Orlu-Gul, Giacomo Fisco, Dipesh Parmar, Hardyal Gill, Catherine Tuleu. A new reconstitutable oral paediatric hydrocortisone solution containing hydroxypropyl- $\beta$ cyclodextrin. Drug Dev Ind Pharm 2013;39:1028-36.

11. www.google.com [Last accessed on 10 Sep 2016]

\section{How to cite this article}

- PS Bhandare, AV Yadav. A review on "dry syrups for paediatrics". Int J Curr Pharm Res 2017;9(1):25-31. 